\title{
An Alternative to Error Correction for SRAM-Like PUFs
}

\author{
Maximilian Hofer and Christoph Boehm \\ Institute of Electronics, Graz University of Technology \\ maximilian.hofer@tugraz.at, christoph.boehm@tugraz.at \\ http://ife.tugraz.at
}

\begin{abstract}
We propose a new technique called stable-PUF-marking as an alternative to error correction to get reproducible (i.e. stable) outputs from physical unclonable functions (PUF). The concept is based on the influence of the mismatch on the stability of the PUF-cells' output. To use this fact, cells providing a high mismatch between their crucial transistors are selected to substantially lower the error rate. To verify the concept, a statistical view to this approach is given. Furthermore, an SRAM-like PUF implementation is suggested that puts the approach into practice.
\end{abstract}

Keywords: Physical Unclonable Functions, SRAM, Pre-Selection.

\section{Introduction}

Due to the widespread use of Smart Cards and radio frequency identification (RFID) devices, the demand for secure identification/authentication and other cryptographic applications is continuously increasing. For this purpose a "fingerprint" of a chip can be useful. Physical unclonable functions (PUFs) provide such an output. In 2001, Pappu et al. introduced the concept of PUFs [1]. In this approach a unique output is produced by evaluating the interference pattern of a transparent optical medium. Unfortunately, due to the way of pattern extraction, Pappu's approach turns out to be quite expensive. In [23], Gassend et al. introduce physical unclonable functions in silicon. The concept utilizes manufacturing process variation to distinguish between different implementations of the same integrated circuit (IC). This is done by measuring the frequency of self-oscillating loop circuits. These frequencies differ slightly between the realizations. However, the chip area is large and the current consumption is high. Another approach is to use the initial values of SRAM cells. 45] shows that there exist SRAM chips which deliver the same start-up value again and again which is the crucial property of a PUF. The best of them deliver an error rate of less than $3 \%$. So it seems that SRAM-like structures are feasible as dedicated PUF-cells 6].

One way to deal with errors in the PUFs' responses is to use error-correction codes (ECC) 7]. Here, redundace is added by storing parity bits during an initialization phase. These bits can be used afterwards to reconstruct the reference

S. Mangard and F.-X. Standaert (Eds.): CHES 2010, LNCS 6225, pp. $335-350,2010$.
(c) International Association for Cryptologic Research 2010 
value. Unfortunately, efficient decoding is difficult. If the error rate is high, the runtime increases strongly 89 . Other methods use statistical data of the fuzziness [10] (i.e. the degree of instability) of the PUF responses. In [10, Maes et. al. read out the response several times to collect data about the stability of the different PUF-cells. An advantage of this Soft Decision Data Helper Algorithm is that the number of PUF-cells can be reduced up to $58.4 \%$. A drawback is that the initialization phase needs a higher number of runs (e.g. 64 in [10]).

In this work we propose an alternative method to deal with unstable PUFcells. The time needed for the read-out phase is reduced due to the fact that further post-processing of the PUF response becomes less complex or even needless depending on the application.

The remainder of the paper is organized as follows. Section 2 describes the idea behind the concept. In Section 3 a statistical analysis is given. Section 4 provides an approach to an implementation in silicon. Finally, section 5 concludes the paper. In the appendix some additional calculations and tables are given.

\section{Idea}

Figure 1 shows a CMOS SRAM-cell that can be used as a PUF-cell. A whole PUF consists of an application dependent number of such cells. We assume that the design and the layout of that PUF-cell are optimized in such a way that the PMOS transistors match and the NMOS transistors mismatch 11 In an SRAM PUF, the output which is defined by the state of OUT after power-up, mainly depends on the threshold voltage $\left(V_{t h}\right)$ mismatch. Assuming identical initial potentials at $O U T$ and $\overline{O U T}$, the mismatch of the NMOS transistors lead to a difference between $i_{1}$ and $i_{2}$ in the two branches of the SRAM cell. If $i_{2}$ is higher than $i_{1}$, the potential at $O U T$ will move towards $V_{S S}$, the potential at $\overline{O U T}$ will move towards $V_{D D}$. If $i_{2}$ is lower than $i_{1}$, the cell behaves the other way round. This behavior at $O U T$ and $\overline{O U T}$ should be an intrinsic property of the cell and should not change over time. If the mismatch is too small, the cell result will be unstable due to noise, temperature shifts, and other shifts in the working point, e.g. caused by changes in $V_{D D}$.

The idea is to select only the stable cells (i.e. those cells providing a high mismatch) to generate the PUF output. Before the PUF is used for the first time, during an initialization phase the stable PUF-cells are detected. These cells are marked. All the other PUF-cells are not used any longer. From now on, only stable PUF-cells generate a stable response.

This gives rise to the question of how to select the stable bits. An intuitive approach is to measure the results of a PUF-cell repeatedly and chose only those cells which always provide the same output. For various reasons this is not practicable. First of all, additional measurements must be done to get useful

\footnotetext{
${ }^{1}$ The mismatch's variance of the transistors can be controlled over the transistor area: Smaller area leads to higher mismatch. This means that the analog designer can influence the variance but not the individual value of the mismatch which defines the PUF-cell output.
} 


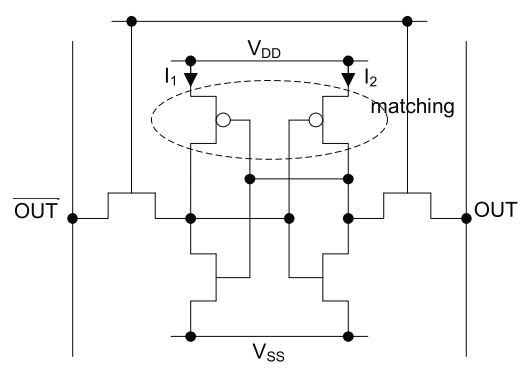

Fig. 1. Common SRAM-cell

statistical data and thus is not feasible for an initial production test flow where measurement time has proportional impact on the product costs. Another problem is that there are cells which show temperature depending behavior. So the initial measurements would have to be done over the whole temperature range. Furthermore, the influence of aging changes the mismatch behavior [5] and could cause additional errors after some time.

Another approach to find unstable PUF-cells is to use the fact that stable cells decide faster 6 . To detect the fast flipping cells, the decision time has to be measured. In figure 2 this concept is illustrated. After a certain time $t_{u s e f u l}$ the

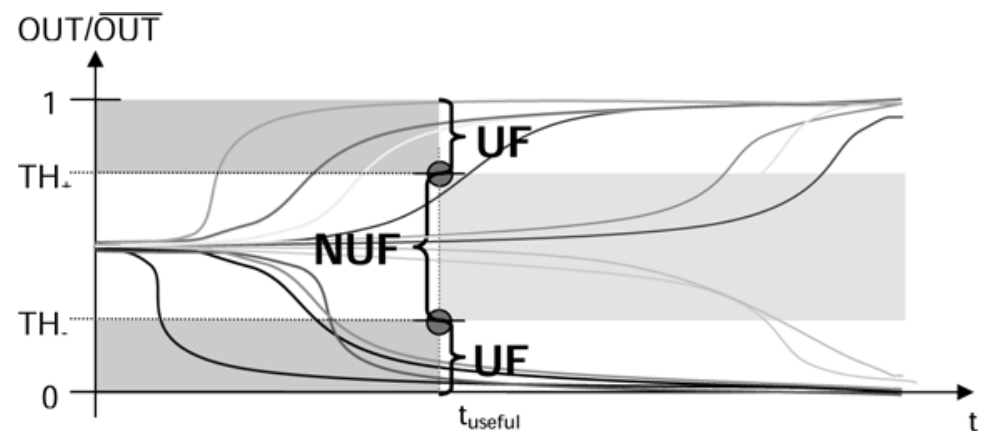

Fig. 2. Measurement of decision time (UF: useful, $N U F$ : not useful)

cells above an upper threshold or under a lower threshold are marked as useful. All other cells which lie between the two thresholds are marked as not useful. Unfortunately, simulations show that the decision time strongly depends on the temperature. Therefore during the initialization phase a constant temperature is necessary to allow the use of an absolute time $t_{\text {use }}$ ul . Another solution to this problem could be to measure the time spans needed to reach a threshold value. The fastest cells are used. Since it may happen that the fastest cells of a chip are still not fast enough to meet the above requirements a stable behavior can not be expected in all cases. 


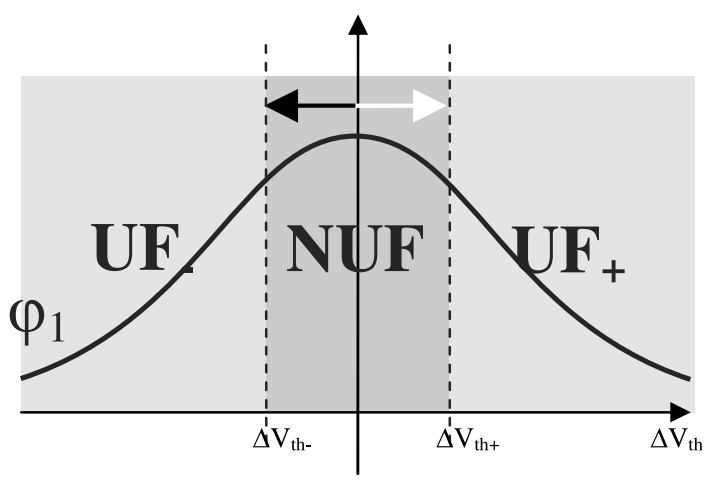

Fig. 3. The mismatch is divided in three classes: The useful PUF-cells with positive mismatch $\left(U F_{+}\right)$, the useful PUF-cells with negative mismatch $\left(U F_{-}\right)$and the not useful PUFs $(N U F)$

The approach we propose in this paper is based on the selection of cells which provide a mismatch that exceeds a certain threshold. In the case of the shown SRAM-PUF, the mismatch of the NMOS transistors must be above such a threshold. In figure 3 , the mismatch $\Delta V_{t h}$ of two transistors is depicted schematically. Here, this distribution is assumed to be Gaussian. A positive and a negative threshold value $\left(\Delta V_{t h+}\right.$ and $\Delta V_{t h-}$, with $\left.\left|\Delta V_{t h+}\right|=\left|\Delta V_{t h-}\right|\right)$ are defined, which is necessary to divide the PUF-cells into three classes: the useful PUF-cells with positive mismatch $\left(U F_{+}\right)$, the useful PUF-cells with negative mismatch $\left(U F_{-}\right)$ and the not useful PUF-cells $(N U F)$. In figure 3, the three sections are depicted. In the middle section, the mismatch is too small to provide a stable behavior. These bits are marked as NUF. The mismatch of the other bits is big enough to provide a stable output. Thus, the threshold value must be chosen correctly to reach an acceptable error rate. The larger the threshold value, the smaller the number of PUF-cells that are marked as stable and the smaller the error rate. Thus, to be able to provide the required number of useful cells, the number of initial PUF-cells has to be adapted to the chosen threshold value. For this reason, the threshold value is a trade-off between the ratio of the useful PUF-cells and all PUF-cells and the error rate.

One method to measure $\Delta V_{t h}$ is to use a common analog to digital converter (ADC). In figure 4 a block diagram is shown. The disadvantage of this approach is the size of the ADC caused by the requirements on it. In order to get a balanced output, the ADC must have a small offset. Furthermore the ADC has to be fast and the result should not depend on the noise of the circuit.

The proposed concept to classify the cells into $U F$ and $N U F$ is to add a systematical $V_{t h}$ offset to the circuit (see figure 7): Two measurements per PUFcell are needed. During the first measurement we add a negative offset. Thus the threshold is set to $V_{t h-}$. During the second measurement we move the threshold to $V_{t h+}$. This is illustrated in figure 3 denoted by the two arrows. The classification can be done as follows: If the mismatch of the transistors exceeds the 


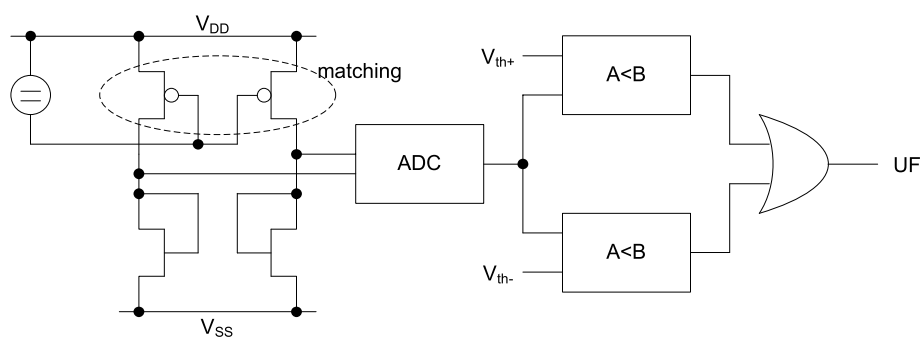

Fig. 4. Measurement of the mismatch using an ADC

threshold, the PUF-cell will provide the same output for both measurements and thus the cell is marked as useful. If the mismatch is too small, the output OUT of the cell will differ for the two measurements. The cell is marked as not useful. Problems will occur if the threshold value is chosen too big. In such a case, only a few or even no cells are marked as useful which can lead to severe problems. On the other hand, if the threshold is chosen too small, disturbances like noise will lead to output errors and make the whole pre-selection process useless.

\section{Modeling and Statistical Aspects}

To analyze the performance of this approach, Monte Carlo simulations are not feasible since the error rate after the pre-selection process (i.e. after the usefulPUF-marking) should be so small that the number of simulation runs to determine the error probability would exceed a tolerable number. So we prefer an analytic method to estimate the performance of the pre-selection process:

For all further analyses we assume that the distribution of the $V_{t h}$ mismatch as well as the distribution of the disturbances (noise, temperature-dependent errors, etc.) is Gaussian 11/2 1314. To determine the effect of the pre-selection process, we need the probability density function (PDF) $f(x)$ and its integral, the cumulative distribution function (CDF) $F(x)$ of a Gaussian:

$$
\begin{gathered}
f(x)=\phi_{\mu, \sigma}(x)=\frac{1}{\sigma \sqrt{2 \pi}} e^{\frac{1}{2}\left(\frac{x-\mu}{\sigma}\right)^{2}} \\
F(x)=\Phi_{\mu, \sigma}(x)=\frac{1}{\sigma \sqrt{2 \pi}} \int_{-\infty}^{x} e^{\frac{1}{2}\left(\frac{x-\mu}{\sigma}\right)^{2}} d x,
\end{gathered}
$$

where $\mu$ is the mean and $\sigma$ the variance of the Gaussian.

If there is no disturbance at the PUF-cell, the cell output will be the same whenever the PUF is read-out. In this case the output would be zero for all PUFcells having a negative $\Delta V_{t h}$ and one for all cells with positive $\Delta V_{t h}$ (see figure 5a). If there are disturbances due to noise, temperature, etc., it may happen that if the mismatch is sufficiently small or the disturbance sufficiently large, the decision is defined by this disturbance. That effect can be seen in figure $5 \mathrm{~b}$ where $\Phi_{2}$ shows the mean output depending on $\Delta V_{t h}$ taking the distribution of the 




(a)

(b)

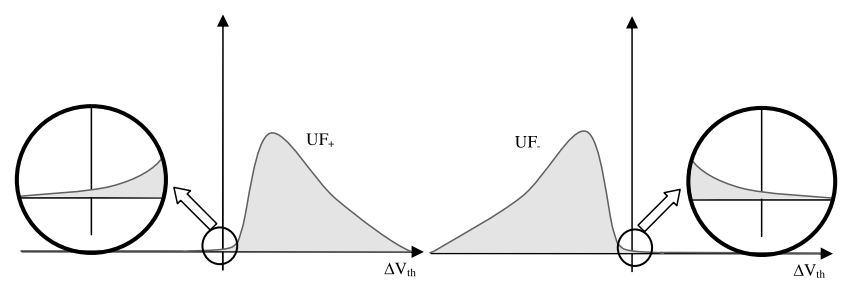

(c)

(d)

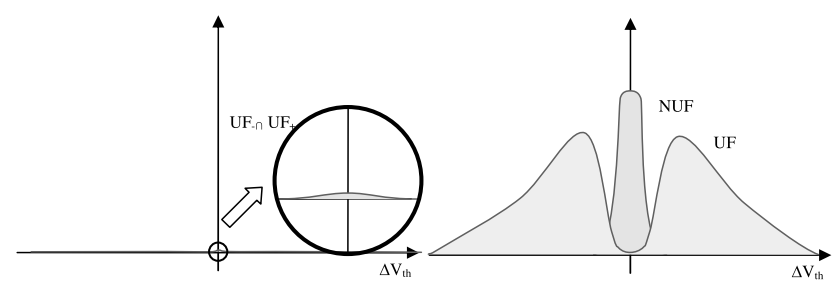

(e)

(f)

Fig. 5. (a) Ideal distributions $\Phi_{1}-\Phi_{4}$.(b) Real distributions $\Phi_{1}-\Phi_{4}$. (c) Useful positive PUF-cells(UF+). (d) Useful negative PUF-cells(UF-). (e) PUF-cells which occur in $U F_{+}$and $U F_{-}$. (f) Useful PUF-cells $(U F)$ and not-useful PUF-cells $(N U F)$.

disturbance into account. At $\Delta V_{t h}=0$ the mean output equals 0.5 . The same curve but biased with the threshold $\Delta V_{t h-}$ and $\Delta V_{t h+}$ depict $\Phi_{4}$ and $\Phi_{3} . \phi_{1}$ is the distribution of the mismatch. After selecting the useful PUF-cells, the error rate can be decreased significantly. Figures $5 \mathrm{c}$ and $5 \mathrm{~d}$ show the product of $\Phi_{3}$ and $\phi_{1}$, and the product of $\left(1-\Phi_{4}\right)$ and $\phi_{1}$ respectively. These curves depict the distribution of being selected as useful including disturbances, the distribution of the $V_{t h}$ mismatch and a certain $V_{t h}$ offset. Hence the figures represent the number of selected PUF-cells. Figure 5e shows those cells that are selected twice, i.e. that are declared to be useful for both offsets. To get correct results these double-selections have to be compensated for in the analysis. Figure $5 f$ shows the distributions of selected and not selected cells.

Since $\sigma_{2}=\sigma_{3}=\sigma_{4}=\sigma, \mu_{1}=\mu_{2}=0$, and $\mu_{3}=-\mu_{4}$ can be assumed, we get the following equation for the number of useful PUF cells $\alpha$ (see appendix): 


$$
\begin{aligned}
\alpha= & -\frac{1}{\sigma_{1} 2 \pi} \int_{\infty}^{-\infty} e^{-\frac{1}{2}\left(\frac{V_{t h}}{\sigma_{1}}\right)^{2}}\left[\frac{1}{\sigma} \int_{-\infty}^{V_{t h}} e^{-\frac{1}{2}\left(\frac{V_{t h}^{\prime}-\mu}{\sigma}\right)^{2}} d V_{t h}^{\prime}+\right. \\
& -\frac{1}{\sigma} \int_{-\infty}^{V_{t h}} e^{-\frac{1}{2}\left(\frac{V_{t h}^{\prime}-\mu}{\sigma}\right)^{2}} d V_{t h}^{\prime}+\frac{2}{\sigma^{2} \sqrt{2 \pi}} \int_{-\infty}^{V_{t h}} e^{-\frac{1}{2}\left(\frac{V_{t h}^{\prime}-\mu}{\sigma}\right)^{2}} d V_{t h}^{\prime} \\
& \left.\cdot \int_{-\infty}^{V_{t h}} e^{-\frac{1}{2}\left(\frac{V_{t h}^{\prime}-\mu}{\sigma}\right)^{2}} d V_{t h}^{\prime}\right] d V_{t h}
\end{aligned}
$$

The error rate $e$ at $\Delta V_{t h}$ can be derived using the following equation (see appendix):

$$
e\left(\Delta V_{t h}\right)=\phi_{1} \Phi_{2}-\phi_{1} \Phi_{2} \Phi_{4}-\phi_{1} \Phi_{2} \Phi_{3} \Phi_{4}+\phi_{1} \Phi_{3}-\phi_{1} \Phi_{3}+\phi_{1} \Phi_{3} \Phi_{4}-\Phi_{2} \phi_{1} \Phi_{3} \Phi_{4},
$$

where all $\phi_{i}$ and $\Phi_{i}$ are evaluated at $\Delta V_{t h}$.

Example. The standard deviation of $\Delta V_{t h}$ is $30 \mathrm{mV}$, the standard deviation of $\phi_{2,3,4}$ equals $6.16 \mathrm{mV}$. This coresponds to an error-rate of $5 \% 2$ In figure 6 the error rate and the ratio of useful PUF-cells $\alpha$ are shown in a diagram. It can be seen that selecting for example the best $50 \%$ can decrease the error rate significantly. A table of different examples is shown in appendix B.

\section{Implementation}

Different circuits are possible to implement the approach described above. One of them is presented. To understand the circuit we consider an ordinary SRAMcell depicted in figure 7. We assume that $P_{1}$ and $P_{2}$ match. Hence, the decision depends on the mismatch of the threshold voltage of $N_{1}$ and $N_{2}$ denoted $\Delta V_{t h}$. To mark the cells as introduced in section 2, we have to add an additional voltage source at the gate of one of the NMOS transistors to provide the bias we need for the threshold (see figure 7a). Since the implementation of such a circuit is difficult, the preferred way is to use its Norton equivalent - a current source - in parallel to one of the NMOS transistors (see figure $7 \mathrm{~b}$ ).

From figure 8, the equivalence of the two circuits can be seen. The characteristics of two different diode-loaded MOSFETS are shown. For the same $V_{G S}$ and different threshold voltages, the amount of current through the transistors will be different. Thus, additional current at one of the branches of the SRAM-cell acts as a mismatch of $V_{t h}$.

The circuit depicted in figure 9 is a practical implementation of the approach. During the first phase $N_{7}$ is switched-off. $N_{3}, N_{4}$ and $P_{1}, P_{2}$ are building a SRAM similar circuit. $N_{2}$ acts as a current limiter for this circuit. The circuit is designed, that the mismatch between $P_{1}$ and $P_{2}$ is small and should not affect the result. Due to the fact that the transistors $N_{3}$ and $N_{4}$ are diode loaded, the

\footnotetext{
${ }^{2}$ We meassured an error-rate of $4 \%$ in a dedicated PUF-cell in the temperature range from $0-80^{\circ} \mathrm{C}$. So this is a rather pessimistic value.
} 


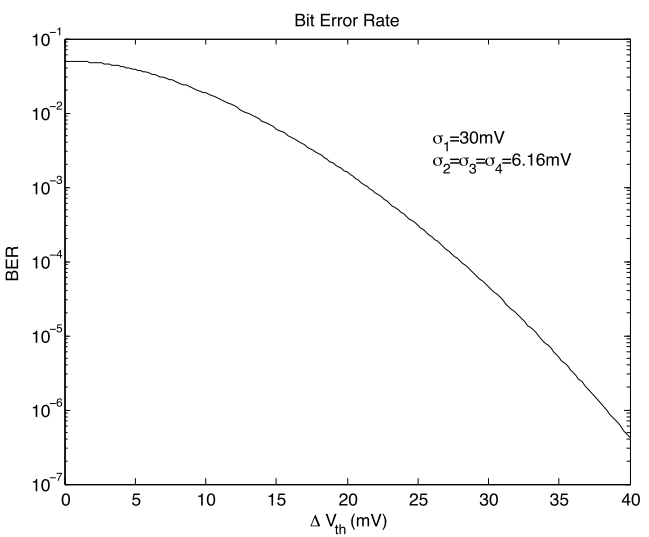

(a)

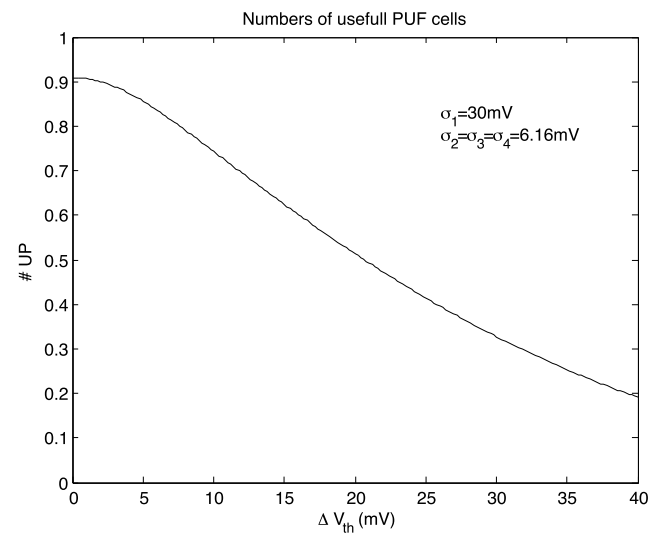

(b)

Fig. 6. a) Error rate $e$. (b) Ratio of useful PUF-cells $\alpha$ against $\mu_{3,4}$.

circuit does not flip as fast as the SRAM depicted in figure 1, During the second phase, $N_{7}$ is switched-on and the circuit flips completely to one direction. The bias transistors which are used for the PUF-cell selection $\left(P_{3}\right.$ and $\left.P_{4}\right)$ are used during the first phase and switched-off during the second phase $\left(P_{7}\right.$ and $P_{8}$ are switched-on; $P_{5}$ and $P_{6}$ are switched-off).

If we want to add a fictive negative offset voltage at the transistor $N_{4}, P_{8}$ is opened and $P_{6}$ is closed. Thus, the transistor $P_{4}$ is in parallel with transistor $P_{2}$. A higher current passes $N_{4}$. The same can be done on the right branch $\left(P_{7}, P_{5}\right.$, $P_{3}$ and $N_{3}$ ). The truth table for the control of the transistors $P_{5}, P_{6}, P_{7}$, and $P_{8}$ is shown in table 1 .

A further improvement of this circuit can be achieved by separating the mismatching transistors $N_{3}$ and $N_{4}$ from the evaluation circuit consisting of the transistors $N_{5}$ to $N_{7}$ and $P_{1}$ to $P_{8}$. Additionally, two transistors are required to connect each cell to the evaluation circuit. So, one PUF-cell consists of only five 


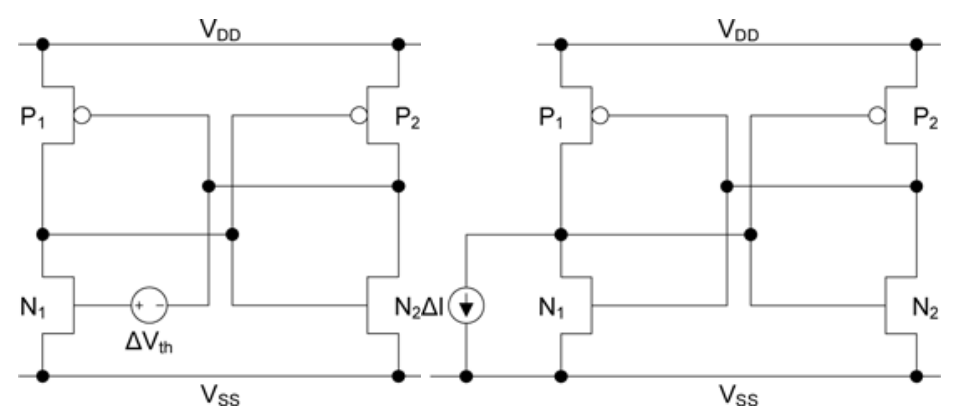

(a)

(b)

Fig. 7. (a) SRAM-cell with additional voltage source at the gate of $N_{1}$; (b) SRAM-cell with additional current source at the drain of $N_{1}$.

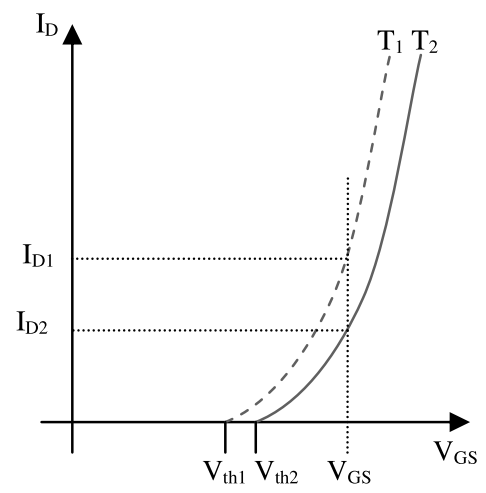

Fig. 8. Characteristics of two MOSFETS having different $V_{t h}$

transistors as depicted in figure 10. The cells can be selected sequentially and evaluated using the same evaluation circuit (i.e. sense amplifier). Thus, the area of one PUF-cell is scaled-down to about the size of a common SRAM-cell. For the particular topology that is about $100 \mathrm{~F}^{2}$ ('minimum featured size').

In such a circuit it could happen that the mismatch of the evaluation circuit influences the decision. Due to this fact, cells using the same evaluation circuit could tend to output the same value. To reduce the influence of an asymmetric sense amplifier, the number of PUF-cells which use the same evaluation logic should be chosen carefully.

The whole structure diagram of the system is depicted in figure 11, There are two modes: One for the initialization phase and another one for the nominal operation. The addresses of the useful cells are stored in a non-volatile memory (NVM). During the initialization phase this memory is filled with data: The outputs of the single PUF-cells after adding both bias currents are compared. If the output stays constant for both bias values, the address of the PUF-cell is written 


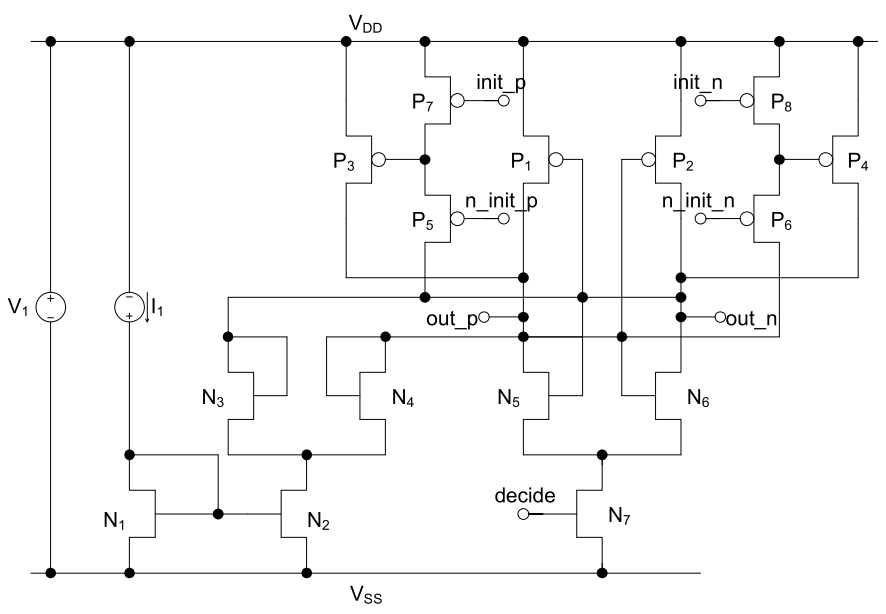

Fig. 9. Implementation example of an SRAM-like PUF with pre-selection transistors $P_{3}$ and $P_{4}$

Table 1. Control of the transistors $P_{5}, P_{6}, P_{7}, P_{8}$ for adding the current bias to the circuit

\begin{tabular}{|c|c|c|c|c|c|c|}
\hline function & $n_{t h}$ & $p_{t h}$ & init $_{p}$ & ninit $_{p}$ & init $_{n}$ & ninit $_{n}$ \\
\hline no threshold & 0 & 0 & 0 & 1 & 0 & 1 \\
p threshold & 0 & 1 & 1 & 0 & 0 & 1 \\
n threshold & 1 & 0 & 0 & 1 & 1 & 0 \\
\hline
\end{tabular}

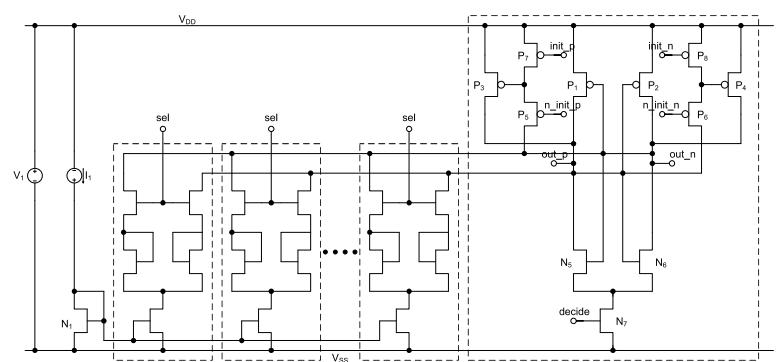

Fig. 10. PUF-cells with shared sense amplifier

into the NVM. The address of the NVM is incremented and the next PUF-cell is tested. This is done until the necessary number of outputs is reached. If not enough useful cells are provided an error occurs and the PUF must be considered to be defect. This indicates that the mismatch between the transistors is too small or that the ratio of required PUF-cells and available PUF-cells is too high. Possible solutions to this problem are to increase the number of PUF-cells or to reduce the upper and lower threshold values $\Delta V_{t h-}$ and $\Delta V_{t h+}$. During the nominal mode, the PUF-cells stored in the NVM are read-out. 


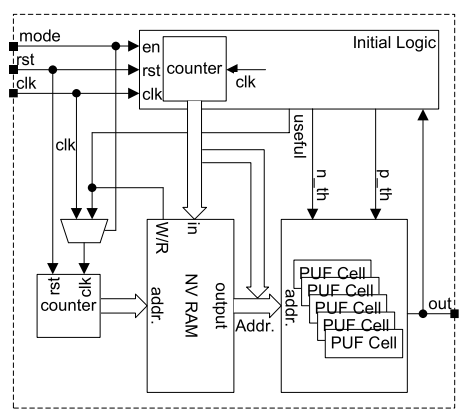

Fig. 11. Structure diagram with initialization logic

\section{Conclusion}

In this paper we introduced a pre-selection process for SRAM-like PUFs which can be implemented with little effort. We demonstrated that error-rates of $10 \mathrm{E}-6$ are achievable. Due to the smaller error rate, using the marking procedure makes post-processing less complex or even unnecessary depending on the application. Hence, the area of the digital part of the circuit can be reduced. Furthermore, the smaller error rate leads to less power consumption and faster read-out. The additional effort caused by the initialization phase is small since the whole process can be done at one temperature and only two read-out cycles are necessary to separate the stable and the unstable PUF-cells.

\section{References}

1. Pappu, R., Recht, R., Taylor, J., Gershenfeld, N.: Physical one-way function. Science 297(5589), 2026-2030 (2002)

2. Gassend, B., Clarke, D., van Dijk, M., Devadas, S.: Silicon physical random functions. In: CCS '02: Proceedings of the 9th ACM conference on Computer and communications. security, pp. 148-160 (2002)

3. Blaise, G., Daihyun, L., Dwaine, C., van Dijk, M., Srinivas, D.: Identification and a2thentication of integrated circuits. Concurrency Computation: Pract. Exper. 16, 1077-1098 (2004)

4. Guajardo, J., Kumar, S.S., Schrijen, G.-J., Tuyls, P.: FPGA Intrinsic PUFs and Their Use for IP Protection. In: Paillier, P., Verbauwhede, I. (eds.) CHES 2007. LNCS, vol. 4727, pp. 63-80. Springer, Heidelberg (2007)

5. Boehm, C., Hofer, M.: Using SRAMs as Physical Unclonable Functions. In: Proceedings of the 17th Austrian Workshop on Microelectronics - Austrochip, pp. 117-122 (2009)

6. Ying, S., Holleman, J., Otis, B.P.: A Digital 1.6 pJ/bit Chip Identification Circuit Using Process Variations. IEEE Journal of Solid-State Circuits 43(1), 69-77 (2008)

7. Dodis, Y.: Fuzzy Extractors: How to Generate Strong Keys from Biometrics and Other Noisy Data. In: Naor, M. (ed.) EUROCRYPT 2007. LNCS, vol. 4515, pp. 523-540. Springer, Heidelberg (2007)

8. Hong, J., Vitterli, M.: Simple Algorithms for BCH Decoding. IEEE Transactions of Communications 43, 2324-2333 (1995) 
9. Boesch, C., Guajardo, J., Sadeghi, A.R., Shokrollahi, J., Tuyls, P.: Efficient Helper Data Key Extractor on FPGAs. In: Oswald, E., Rohatgi, P. (eds.) CHES 2008. LNCS, vol. 5154, pp. 181-197. Springer, Heidelberg (2008)

10. Maes, R., Tuyls, P., Verbauwhede, I.: Low-Overhead Implementation of a Soft Decision Helper Data Algorithm for SRAM PUFs. In: Clavier, C., Gaj, K. (eds.) CHES 2009. LNCS, vol. 5747, pp. 332-347. Springer, Heidelberg (2009)

11. Pelgrom, M., Duinmaijer, A., Welbers, A.: Matching Properties of MOSTransistors. IEEE Journal of Solid-State Circuits 24, 1433-1440 (1989)

12. Pelgrom, M.J.M., Tuinhout, H.P., Vertregt, M.: Transistor matching in analog CMOS applications. In: International Electron Devices Meeting, IEDM '98 Technical Digest., pp. 915-918 (1998)

13. Mizuno, T., Okumtura, J., Toriumi, A.: Experimental study of threshold voltage fluctuation due to statistical variation of channel dopant number in MOSFET's. IEEE Transactions on Electron Devices 41, 2216-2221 (1994)

14. Tsividis, Y.: The MOS Transistor. Oxford University Pres, New York (1999)

\section{A Calculations}

Ratio of Useful PUF-Cells $\alpha$ : Partial probability of occurrence of selected PUF cells depending on $\Delta V_{t h}$ (see figure $7(\mathrm{c})$ and $\left.7(\mathrm{~d})\right)^{3}$

$$
\begin{gathered}
U F_{+}=\phi_{1} \Phi_{3} \\
U F_{-}=\phi_{1}\left(1-\Phi_{4}\right)
\end{gathered}
$$

Probability of occurrence PUF-cells being selected twice depending on $\Delta V_{t h}$ (see figure $7(\mathrm{e}))$ :

$$
U F_{+} \cap U F_{-}=\phi_{1} \Phi_{3}\left(1-\Phi_{4}\right)
$$

Total probability of occurrence depending on $\Delta V_{t h}$ :

$$
\begin{aligned}
U F & =U F_{+}+U F_{-}-2\left(U F_{+} \cap U F_{-}\right)= \\
& =\phi_{1}\left[\Phi_{3}+\left(1-\Phi_{4}\right)-2 \Phi_{3}\left(1-\Phi_{4}\right)\right]= \\
& =\phi_{1}\left[1-\Phi_{4}-\Phi_{3}+2 \Phi_{3} \Phi_{4}\right]
\end{aligned}
$$

From $U F$ the ratio of useful PUF-cells $\alpha$ can be determined:

$$
\begin{aligned}
\alpha= & \int_{\infty}^{-\infty} U F d V_{t h}= \\
= & \int_{\infty}^{-\infty} \frac{1}{\sigma_{1} \sqrt{2 \pi}} e^{-\frac{1}{2}\left(\frac{V_{t h}-\mu_{1}}{\sigma_{1}}\right)^{2}}\left[1-\frac{1}{\sigma_{4} \sqrt{2 \pi}} \int_{-\infty}^{V_{t h}} e^{-\frac{1}{2}\left(\frac{V_{t h}^{\prime}-\mu_{4}}{\sigma_{4}}\right)^{2}} d V_{t h}^{\prime}+\right. \\
& -\frac{1}{\sigma_{3} \sqrt{2 \pi}} \int_{-\infty}^{V_{t h}} e^{-\frac{1}{2}\left(\frac{V_{t h}^{\prime}-\mu_{3}}{\sigma_{3}}\right)^{2}} d V_{t h}^{\prime}+\frac{2}{\sigma_{3} \sqrt{2 \pi}} \int_{-\infty}^{V_{t h}} e^{-\frac{1}{2}\left(\frac{V_{t h}^{\prime}-\mu_{3}}{\sigma_{3}}\right)^{2}} d V_{t h}^{\prime} .
\end{aligned}
$$

\footnotetext{
${ }^{3}$ The results of this section depend on the threshold values $V_{t h+}$ and $V_{t h-}$.
} 


$$
\begin{aligned}
& \left.\cdot \frac{1}{\sigma_{4} \sqrt{2 \pi}} \int_{-\infty}^{V_{t h}} e^{-\frac{1}{2}\left(\frac{V_{t h}^{\prime}-\mu_{4}}{\sigma_{4}}\right)^{2}} d V_{t h}^{\prime}\right] d V_{t h}= \\
= & 1-\frac{1}{\sigma_{1} 2 \pi} \int_{\infty}^{-\infty} e^{-\frac{1}{2}\left(\frac{V_{t h}-\mu_{1}}{\sigma_{1}}\right)^{2}\left[\frac{1}{\sigma_{4}} \int_{-\infty}^{V_{t h}} e^{-\frac{1}{2}\left(\frac{V_{t h}^{\prime}-\mu_{4}}{\sigma_{4}}\right)^{2}} d V_{t h}^{\prime}+\right.} \\
& -\frac{1}{\sigma_{3}} \int_{-\infty}^{V_{t h}} e^{-\frac{1}{2}\left(\frac{V_{t h}^{\prime}-\mu_{3}}{\sigma_{3}}\right)^{2}} d V_{t h}^{\prime}+\frac{2}{\sigma_{3} \sigma_{4} \sqrt{2 \pi}} \int_{-\infty}^{V_{t h}} e^{-\frac{1}{2}\left(\frac{V_{t h}^{\prime}-\mu_{3}}{\sigma_{3}}\right)^{2}} d V_{t h}^{\prime} . \\
& \left.\cdot \int_{-\infty}^{V_{t h}} e^{-\frac{1}{2}\left(\frac{V_{t h}^{\prime}-\mu_{4}}{\sigma_{4}}\right)^{2}} d V_{t h}^{\prime}\right] d V_{t h}
\end{aligned}
$$

In general we can assume that $\sigma_{2}=\sigma_{3}=\sigma_{4}=\sigma, \mu_{1}=\mu 2=0$, and $\mu_{3}=-\mu_{4}$. Then $\alpha$ becomes:

$$
\begin{aligned}
\alpha= & -\frac{1}{\sigma_{1} 2 \pi} \int_{\infty}^{-\infty} e^{-\frac{1}{2}\left(\frac{V_{t h}}{\sigma_{1}}\right)^{2}}\left[\frac{1}{\sigma} \int_{-\infty}^{V_{t h}} e^{-\frac{1}{2}\left(\frac{V_{t h}^{\prime}-\mu}{\sigma}\right)^{2}} d V_{t h}^{\prime}+\right. \\
& -\frac{1}{\sigma} \int_{-\infty}^{V_{t h}} e^{-\frac{1}{2}\left(\frac{V_{t h}^{\prime}-\mu}{\sigma}\right)^{2}} d V_{t h}^{\prime}+\frac{2}{\sigma^{2} \sqrt{2 \pi}} \int_{-\infty}^{V_{t h}} e^{-\frac{1}{2}\left(\frac{V_{t h}^{\prime}-\mu}{\sigma}\right)^{2}} d V_{t h}^{\prime} . \\
& \left.\cdot \int_{-\infty}^{V_{t h}} e^{-\frac{1}{2}\left(\frac{V_{t h}^{\prime}-\mu}{\sigma}\right)^{2}} d V_{t h}^{\prime}\right] d V_{t h}
\end{aligned}
$$

Ratio of Not-Useful PUF-Cells $\beta$ : To verify the result of $\alpha$, the ratio $\beta$ of not selected PUF-cells is determined as well:

$$
\begin{gathered}
\beta=\int_{\infty}^{-\infty} N U F d V_{t h} \\
N U F=\phi_{1}\left[\left(1-\Phi_{3}\right)\left(1-\left(1-\Phi_{4}\right)+\Phi_{3}\left(1-\Phi_{4}\right)\right)\right] \\
\left.=\phi_{1}\left[\left(1-\Phi_{3}\right)\left(\Phi_{4}\right)+\Phi_{3}\left(1-\Phi_{4}\right)\right)\right] \\
\left.\left.=\phi_{1}\left[\Phi_{4}-\Phi_{4} \Phi_{3}+\Phi_{3}-\Phi_{4} \Phi_{3}\right)\right)\right] \\
\left.\left.=\phi_{1}\left[\Phi_{4}+\Phi_{3}-2 \Phi_{4} \Phi_{3}\right)\right)\right] \\
\beta=\int_{\infty}^{-\infty} \frac{1}{\sigma_{1} \sqrt{2 \pi}} e^{-\frac{1}{2}\left(\frac{V_{t h}-\mu_{1}}{\sigma_{1}}\right)^{2}} \frac{1}{\sigma_{3} \sqrt{2 \pi}} \int_{-\infty}^{V_{t h}} e^{-\frac{1}{2}\left(\frac{V_{t h}^{\prime}-\mu_{3}}{\sigma_{3}}\right)^{2}} d V_{t h}^{\prime}+ \\
+\frac{1}{\sigma_{4} \sqrt{2 \pi}} \int_{-\infty}^{V_{t h}} e^{-\frac{1}{2}\left(\frac{V_{t h}^{\prime}-\mu_{4}}{\sigma_{4}}\right)^{2}} d V_{t h}^{\prime}-\frac{2}{\sigma_{3} \sqrt{2 \pi}} \int_{-\infty}^{V_{t h}} e^{-\frac{1}{2}\left(\frac{V_{t h}^{\prime}-\mu_{3}}{\sigma_{3}}\right)^{2}} d V_{t h}^{\prime} \\
\frac{1}{\left.\sigma_{4} \sqrt{2 \pi} \int_{-\infty}^{V_{t h}} e^{-\frac{1}{2}\left(\frac{V_{t h}^{\prime}-\mu_{4}}{\sigma_{4}}\right)^{2}} d V_{t h}^{\prime}\right]} d V_{t h}=
\end{gathered}
$$




$$
\begin{aligned}
= & \frac{1}{\sigma_{1} 2 \pi} \int_{\infty}^{-\infty} e^{-\frac{1}{2}\left(\frac{V_{t h}-\mu_{1}}{\sigma_{1}}\right)^{2}}\left[\frac{1}{\sigma_{3}} \int_{-\infty}^{V_{t h}} e^{-\frac{1}{2}\left(\frac{V_{t h}^{\prime}-\mu_{3}}{\sigma_{3}}\right)^{2}} d V_{t h}^{\prime}+\right. \\
& +\frac{1}{\sigma_{4}} \int_{-\infty}^{V_{t h}} e^{-\frac{1}{2}\left(\frac{V_{t h}^{\prime}-\mu_{4}}{\sigma_{4}}\right)^{2}} d V_{t h}^{\prime}-\frac{2}{\sigma_{3} \sigma_{4} \sqrt{2 \pi}} \int_{-\infty}^{V_{t h}} e^{-\frac{1}{2}\left(\frac{V_{t h}^{\prime}-\mu_{3}}{\sigma_{3}}\right)^{2}} d V_{t h}^{\prime} . \\
& \left.\cdot \int_{-\infty}^{V_{t h}} e^{-\frac{1}{2}\left(\frac{V_{t h}^{\prime}-\mu_{4}}{\sigma_{4}}\right)^{2}} d V_{t h}^{\prime}\right] d V_{t h}
\end{aligned}
$$

In general we can assume that $\sigma_{2}=\sigma_{3}=\sigma_{4}=\sigma, \mu_{1}=\mu 2=0$, and $\mu_{3}=-\mu_{4}$. Thus, $\beta$ becomes:

$$
\begin{aligned}
\beta= & \frac{1}{\sigma_{1} \sigma 2 \pi} \int_{\infty}^{-\infty} e^{-\frac{1}{2}\left(\frac{V_{t h}}{\sigma_{1}}\right)^{2}}\left[\int_{-\infty}^{V_{t h}} e^{-\frac{1}{2}\left(\frac{V_{t h}^{\prime}-\mu}{\sigma}\right)^{2}} d V_{t h}^{\prime}+\right. \\
& +\int_{-\infty}^{V_{t h}} e^{-\frac{1}{2}\left(\frac{V_{t h}^{\prime}-\mu_{4}}{\sigma_{4}}\right)^{2}} d V_{t h}^{\prime}-\frac{2}{\sigma} \int_{-\infty}^{V_{t h}} e^{-\frac{1}{2}\left(\frac{V_{t h}^{\prime}-\mu}{\sigma}\right)^{2}} d V_{t h}^{\prime} \\
& \left.\cdot \int_{-\infty}^{V_{t h}} e^{-\frac{1}{2}\left(\frac{V_{t h}^{\prime}-\mu}{\sigma}\right)^{2}} d V_{t h}^{\prime}\right] d V_{t h}
\end{aligned}
$$

Check: $1=\alpha+\beta$

Estimation of the Error Rate e: An error occurs, if one of the PUF-cells which were marked useful provides the wrong output. Like the total ratio of selected PUFs, the total error $e\left(\Delta V_{t h}\right)$ is the sum of the two partial errors $e_{-}\left(\Delta V_{t h}\right)$ and $e_{+}\left(\Delta V_{t h}\right)$. The following errors are evaluated at a certain $e\left(\Delta V_{t h}\right)$ :

$$
\begin{aligned}
e_{-}\left(\Delta V_{t h}\right) & =\frac{1}{\alpha} \Phi_{2}\left[U F_{-}-\left(U F_{+} \cap U F_{-}\right)\right]= \\
& =\frac{1}{\alpha} \Phi_{2}\left[\phi_{1}\left(1-\Phi_{4}\right)-\phi_{1} \Phi_{3}\left(1-\Phi_{4}\right)\right] \\
e_{+}\left(\Delta V_{t h}\right)= & \frac{1}{\alpha}\left(1-\Phi_{2}\right)\left[U F_{+}-\left(U F_{+} \cap U F_{-}\right)\right]= \\
= & \frac{1}{\alpha}\left(1-\Phi_{2}\right)\left[\phi_{1} \Phi_{3}-\phi_{1} \Phi_{3}\left(1-\Phi_{4}\right)\right],
\end{aligned}
$$

where $\frac{1}{\alpha}$ is a normalization factor.

$$
\begin{aligned}
e\left(\Delta V_{t h}\right)= & e_{+}\left(\Delta V_{t h}\right)+e_{-}\left(\Delta V_{t h}\right)= \\
= & \frac{1}{\alpha}\left(1-\Phi_{2}\right)\left[\phi_{1} \Phi_{3}-\phi_{1} \Phi_{3}\left(1-\Phi_{4}\right)\right]+ \\
& +\frac{1}{\alpha} \Phi_{2}\left[\phi_{1}\left(1-\Phi_{4}\right)-\phi_{1} \Phi_{3}\left(1-\Phi_{4}\right)\right]= \\
= & \frac{1}{\alpha}\left\{\left[\phi_{1} \Phi_{2}-\phi_{1} \Phi_{2} \Phi_{4}-\phi_{1} \Phi_{2} \Phi_{3}+\phi_{1} \Phi_{2} \Phi_{3} \Phi_{4}\right]+\right.
\end{aligned}
$$




$$
\begin{aligned}
& \left.+\left[\phi_{1} \Phi_{3}-\phi_{1} \Phi_{3}\left(1-\Phi_{4}\right)\right]-\Phi_{2} \phi_{1} \Phi_{3}+\Phi_{2} \phi_{1} \Phi_{3}\left(1-\Phi_{4}\right)\right\}= \\
= & \frac{1}{\alpha}\left\{\phi_{1} \Phi_{2}-\phi_{1} \Phi_{2} \Phi_{4}-\phi_{1} \Phi_{2} \Phi_{3} \Phi_{4}+\phi_{1} \Phi_{3}-\phi_{1} \Phi_{3}+\phi_{1} \Phi_{3} \Phi_{4}-\right. \\
& \left.-\Phi_{2} \phi_{1} \Phi_{3}+\Phi_{2} \phi_{1} \Phi_{3}-\Phi_{2} \phi_{1} \Phi_{3} \Phi_{4}\right\}
\end{aligned}
$$

Table 2. Examples for the error rate $e$ and the ratio of useful PUF-cells $\alpha$. ${ }^{*}$ The number in the brackets shows the BER without any pre-selection.

\begin{tabular}{|c|c|c|c|c|c|}
\hline num & $\sigma_{1}$ & $\sigma_{2}, \sigma_{3}, \sigma_{4}$ & $\mu_{3}=\mu_{4}$ & $\alpha$ & $e$ \\
\hline 1 & $30 \mathrm{mV}$ & $1 \mathrm{mV}\left(\approx 0.7 \%^{*}\right)$ & $5 \mathrm{mV}$ & 0.8677 & $2.19 \mathrm{E}-06$ \\
2 & $30 \mathrm{mV}$ & $1 \mathrm{mV}\left(\approx 0.7 \%^{*}\right)$ & $10 \mathrm{mV}$ & 0.7390 & $\lceil 1 \mathrm{e}-12$ \\
3 & $30 \mathrm{mV}$ & $2 \mathrm{mV}\left(\approx 1.5 \%^{*}\right)$ & $10 \mathrm{mV}$ & 0.7394 & $5.09 \mathrm{E}-6$ \\
4 & $30 \mathrm{mV}$ & $2 \mathrm{mV}\left(\approx 1.5 \%^{*}\right)$ & $20 \mathrm{mV}$ & 0.5059 & $\lceil 1 \mathrm{e}-12$ \\
5 & $30 \mathrm{mV}$ & $5 \mathrm{mV}\left(\approx 4 \%^{*}\right)$ & $10 \mathrm{mV}$ & 0.7422 & 0.0087 \\
6 & $30 \mathrm{mV}$ & $5 \mathrm{mV}\left(\approx 4 \%^{*}\right)$ & $20 \mathrm{mV}$ & 0.5108 & $2.39 \mathrm{E}-4$ \\
7 & $30 \mathrm{mV}$ & $5 \mathrm{mV}\left(\approx 4 \%^{*}\right)$ & $40 \mathrm{mV}$ & 0.1884 & $1.03 \mathrm{E}-9$ \\
\hline
\end{tabular}

Table 3. Numeric examples of the error rate $e$ and the ratio of useful PUF-cells $\alpha$ in dependence of $\mu_{3,4}\left(\sigma_{1}=30 \mathrm{mV}, \sigma_{2,3,4}=6,16 \mathrm{mV}\right)$. Without any pre-selection $\left(\mu_{3,4}=0 \mathrm{mV}\right)$ we get an error-rate of about $5 \%$.

\begin{tabular}{|c|c|c||c|c|c|}
\hline$\mu_{3,4}(\mathrm{mV})$ & $e$ & $\alpha$ & $\mu_{3,4}(\mathrm{mV})$ & $e$ & $\alpha$ \\
\hline 0 & $4.9965 \mathrm{E}-2$ & 0.909 & 26 & $2.1295 \mathrm{E}-4$ & 0.396 \\
1 & $4.9435 \mathrm{E}-2$ & 0.907 & 27 & $1.4701 \mathrm{E}-4$ & 0.378 \\
2 & $4.7882 \mathrm{E}-2$ & 0.9 & 28 & $1.0037 \mathrm{E}-4$ & 0.361 \\
3 & $4.5412 \mathrm{E}-2$ & 0.889 & 29 & $6.7765 \mathrm{E}-5$ & 0.344 \\
4 & $4.2189 \mathrm{E}-2$ & 0.874 & 30 & $4.5242 \mathrm{E}-5$ & 0.327 \\
5 & $3.8415 \mathrm{E}-2$ & 0.856 & 31 & $2.9867 \mathrm{E}-5$ & 0.311 \\
6 & $3.4307 \mathrm{E}-2$ & 0.836 & 32 & $1.9495 \mathrm{E}-5$ & 0.296 \\
7 & $3.0078 \mathrm{E}-2$ & 0.814 & 33 & $1.2581 \mathrm{E}-5$ & 0.281 \\
8 & $2.5917 \mathrm{E}-2$ & 0.791 & 34 & $8.027 \mathrm{E}-6$ & 0.267 \\
9 & $2.1971 \mathrm{E}-2$ & 0.767 & 35 & $5.0631 \mathrm{E}-6$ & 0.253 \\
10 & $1.8347 \mathrm{E}-2$ & 0.743 & 36 & $3.1571 \mathrm{E}-6$ & 0.24 \\
11 & $1.5109 \mathrm{E}-2$ & 0.719 & 37 & $1.9460 \mathrm{E}-6$ & 0.227 \\
12 & $1.2282 \mathrm{E}-2$ & 0.695 & 38 & $1.1858 \mathrm{E}-6$ & 0.215 \\
13 & $9.8629 \mathrm{E}-3$ & 0.671 & 39 & $7.1416 \mathrm{E}-7$ & 0.203 \\
14 & $7.8298 \mathrm{E}-3$ & 0.648 & 40 & $4.2516 \mathrm{E}-7$ & 0.192 \\
15 & $6.1474 \mathrm{E}-3$ & 0.624 & 41 & $2.5017 \mathrm{E}-7$ & 0.181 \\
16 & $4.7749 \mathrm{E}-3$ & 0.601 & 42 & $1.4549 \mathrm{E}-7$ & 0.17 \\
17 & $3.6698 \mathrm{E}-3$ & 0.579 & 43 & $8.363 \mathrm{E}-8$ & 0.16 \\
18 & $2.7909 \mathrm{E}-3$ & 0.557 & 44 & $4.7509 \mathrm{E}-8$ & 0.151 \\
19 & $2.1004 \mathrm{E}-3$ & 0.535 & 45 & $2.6674 \mathrm{E}-8$ & 0.142 \\
20 & $1.5641 \mathrm{E}-3$ & 0.514 & 46 & $1.4800 \mathrm{E}-8$ & 0.133 \\
21 & $1.1525 \mathrm{E}-3$ & 0.493 & 47 & $8.1156 \mathrm{E}-9$ & 0.125 \\
22 & $8.4015 \mathrm{E}-4$ & 0.473 & 48 & $4.3978 \mathrm{E}-9$ & 0.117 \\
23 & $6.0592 \mathrm{E}-4$ & 0.453 & 49 & $2.3550 \mathrm{E}-9$ & 0.11 \\
24 & $4.3229 \mathrm{E}-4$ & 0.433 & 50 & $1.2462 \mathrm{E}-9$ & 0.103 \\
25 & $3.0507 \mathrm{E}-4$ & 0.414 & & & \\
\hline & \multicolumn{5}{|l|}{} \\
\end{tabular}


To get the error $e$ over all $e\left(\Delta V_{t h}\right), e\left(\Delta V_{t h}\right)$ has to be integrated over all $\Delta V_{t h}$ :

$$
e=\int_{\infty}^{-\infty} e\left(\Delta V_{t h}\right) d \Delta V_{t h}
$$

\section{B Numerical Examples}

Table 2 shows some numeric examples for $\alpha$ and $e$. The number of useful PUFcells depends mainly on the ratio $\frac{\sigma_{1}}{\mu_{3,4}}$. The main factors for the error are $\sigma_{2,3,4}$ and $\mu_{3,4} . \sigma_{1}$ influences the error rate only marginally. Table 3 shows $e$ in dependence of $\mu_{3,4}$. 